\title{
Friction in nature
}

\author{
T. Liskiewicz ${ }^{1}$, A. Morina ${ }^{1,2}$ \& A. Neville ${ }^{1}$ \\ ${ }^{1}$ Institute of Engineering Thermofluids Surfaces \& Interfaces, \\ School of Mechanical Engineering, University of Leeds, UK \\ ${ }^{2}$ School of Process, Environmental and Materials Engineering (SPEME), \\ University of Leeds, UK
}

\begin{abstract}
Nature has found a number of ways to efficiently tackle friction problems. It has solutions to provide ultra low friction in the case of lubricated systems, ultra high friction in the case of adhesives or in some cases even controlled adaptable friction performance. Frictional surfaces can be found on different scales in nature from a nanometre scale to a macro scale. From a wide variety of natural systems synovial joints have probably attracted more research attention than other systems and this is fully justified, as they are examples of ideal synergy between lubrication process and materials technology. Other examples include shark skin riblets for drag reduction, hierarchical structures for maximum adhesion or mucus and slime for an improved slip. In this contribution a summary of natural frictional systems is presented. Several examples of the successful use of biomimicry for the manipulation of the interface to provide the desired functions are presented here. Finally directions of further exploitation of a biomimetic approach in tribology are discussed.

Keywords: friction, biomimetics, surface engineering, hierarchical structures, drag reduction, adhesion, nano-technology, lubrication.
\end{abstract}

\section{Introduction}

Natural systems attract a lot of attention from engineers, not only due to their sophisticated beauty but mainly because of their high efficiency and durability. Biomimetics is an emerging field of research and its benefits have been found in a number of areas from architecture, robotics to materials science and many more [1]. A biomimetic approach benefits from the fact that nature is regarded as 
an extremely effective problem solver. Natural systems have been evolved and developed for billions of years and so the solutions applied by nature are simple, efficient and very often perform under severe conditions.

One of the disciplines that can benefit from biomimetics is tribology, which deals with friction, lubrication and wear processes in engineering systems [2]. To date little biomimetics research has been directed towards tribology in terms of transferring technologies from biological systems into engineering applications. The field of tribology is however just a tool to observe and interpret the phenomena at the frictional interface and when it comes to the problem solution surface engineering is a relevant area. Hence tribology and surface engineering has to come together, firstly to recognise and interpret the frictional problem and secondly to provide a suitable solution for low friction, improved wear resistance, enhanced adhesion, reduced drag or tailored lubrication.

When looking at frictional systems in nature, two cases can be distinguished: (i) systems which have been evolved for millions of years to adapt to unique frictional requirements, and (ii) systems which have an interesting frictional properties from an engineering point of view but their evolution have not been driven by the frictional requirements. In this paper the review of natural systems with unique frictional properties follows that systematisation. The focus is to consider biomimetics, and in particular mimicking the natural systems, as a means of making advances towards more effective engineering tribological systems.

\section{Animal world}

Adaptation is an unique feature of living organisms, which enable change of structural, behavioural or physiological conditions to cope with external stimuli. Some natural systems have been adapted and improved through the evolution for millions of years to have optimal frictional properties. This mainly relates to the animals as the evolution allow them to survive and successfully operate in their natural habitat. In this section the focus is on four examples to illustrate (i) mechanisms of lubricated synovial joint contact, (ii) function of mucus and (iii) hierarchical structures for maximum adhesion.

\subsection{Lubricated synovial joint contact}

Synovial joints have probably attracted more research attention than other systems and this is fully justified as they are examples of ideal synergy between lubrication process and materials technology. The major elements of the natural synovial joint are shown in Figure 1a [3]. Articular cartilage (AC) is a soft porous composite material. The main constituents of AC are collagen, proteoglycans and water. Collagen and proteoglycans in the cartilage form interpenetrating networks that create a strong solid matrix and there is around $80 \%$ water in the cartilage. The cartilage is bonded to the bone and behaves as a thin-layer cushion contact. As shown in Figure 1b, the articular cartilage is reported to have three main layers depending on the fibre orientation [4]. The 
major non-collagenous components of the solid phase of AC are proteoglycan macromolecules. The concentration of proteoglycans is lowest near the AC surface and increases with depth. The proteoglycan macromolecules consist of a protein core in which 50-100 glycosaminoglycans chains are bonded to form a bottlebrush-like structure. These structures are then aggregated to a backbone of hyaluronic acid, Figure 1c, to form a macromolecule with a weight up to 200 million and a length of approximately $2 \mu \mathrm{m}$ [5].
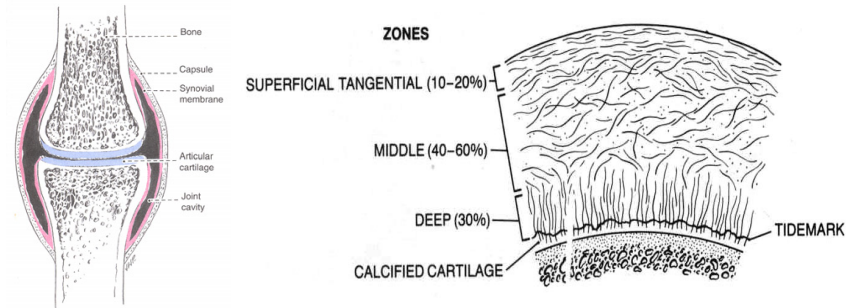

Figure 1: (a) Synovial joint capsule [3]; (b) internal structure of the articular cartilage [4]; c) proteoglycan structures aggregated to a backbone of hyaluronic acid [5].

Proteoglycans are negatively charged and attract the hydrogen atoms of the water molecules, hydrating the zone where there are proteoglycans. Absorption of water from the synovial joints results in swelling of the collagen fibrils [6]. The compressive properties of cartilage are provided partly by the proteoglycans that resist compression because glycosaminoglycans chains repulse each other due to their negative charges [7]. These characteristics of proteoglycans, water attraction and repulsion from each other, provide the viscoelastic properties of articular cartilage, very important properties for effective lubrication.

Critical to the successful long-term tribological function of synovial joints, besides the mechanical properties of $\mathrm{AC}$, is also the nature of the synovial fluid. Synovial fluid is essentially a dialysate of blood plasma with chief constituent being water and containing: long chain protein molecules, hyaluronic acid and phospholipids. It has been suggested [8] that interstitial fluid pressurization is a primary mechanism in the regulation of the friction response of articular cartilage. By supporting the majority of the load transmitted across the contact interface, the interstitial pressurization reduces the load supported by the contacting collagen-proteoglycan matrix and opposing surface, considerably reducing the frictional force relative to the total contact force.

The lubrication mechanism in the joint appears to consist of a fluid in contact with sliding surfaces coated with an oligolamellar lining of Surface Active PhosphoLipids (SAPL) [9]. As the outermost layer, this lining provides boundary lubrication and imparts the hydrophobicity characteristics of these surfaces when rinsed free of synovial fluid, which appears to contain a wetting agent to promote hydrodynamic lubrication. Researchers have speculated that SAPL is the boundary lubricant found wherever tissues need to slide over each other, also acting as an antistick agent [10]. 


\subsection{Function of mucus}

Mucus is a slippery secretion of the lining of the mucous membranes and it is a viscous colloid containing water and some additives in the form of proteins. Depending on the composition of those additives, mucus can provide different frictional properties to the natural system.

In case of snails for example, the foot secrets mucus, containing water and 3 4 per cent glycoprotein [11], allows those creatures to move by constantly maintaining a thin film between snail foot sole and the ground. It has been found that mucus has some unique shear-thinning properties [12], which allow them to use a minimum amount of fluid to crawl. It has a great meaning since making mucus requires an energy, so by producing less mucus they can use more the energy to power their muscles and move faster. In the same time presence of mucus at the interface provides enough adhesion for a snail to travel across vertical surfaces.

Another example refers to tree frogs, which as geckos do - use microscopic spatula pads to provide adhesion but additionally employ mucus [13]. They have an unique ability to stick to vertical or even ceiling-type surfaces and can detach their pads easily when they need to move. The role of mucus is to provide wet adhesion by preventing the friction between frog's feet and inclined surface. They have also developed a special ability to balance their body in such a way so that they always keep the minimum angle of their toes to the surface, which prevents detachment.

It has been observed that mucus can also contribute to the drag reduction of fish [14]. All fish species are covered with mucus that supports many aspects of their existence, from thermal regulation, through disease resistance to locomotion [15]. In some cases, after comparison of velocity profiles for water flow over a fish body it has been found that slime secretion can reduce drag by $50-60 \%$ [16]. The mechanism behind that drop is believed to be related to the decrease of viscosity of water at the mucus/water interface. The action of mucus is however not the only factor responsible for low drag properties of fish and more detailed analysis is necessary to explain that phenomenon.

\subsection{Hierarchical structures for maximum adhesion}

Adhesion is a specific tribological case where friction between two surfaces is optimised to provide a bonding force. Adhesion in natural systems is achieved in different ways, depending mainly on the evolutionary processes. Studying the natural systems, one key feature is the hierarchical organisation of the natural materials. Indeed, all materials are hierarchically built starting from atoms, molecules, assemblages of molecular units, combination of different molecular units etc. What is unique for natural materials is that the hierarchical structure of the material is developed to enable different functionalities. Performance of natural materials is highly depended on the hierarchical organisation over a large range of length scales [17]. Most prominent examples are the hierarchical structure of wood, bone, abalone shell etc. 
In terms of adhesion, one interesting example of the hierarchical structure which enables high adhesion is the hairy attachment structure in geckos. High adhesion of gecko's feet enables them to run across vertical wall as well as ceiling. Detailed research has shown that gecko adhesion to surfaces relies on foot pads composed of millions of tiny branching hairs [18,19]. Gecko's toes contain setal arrays consisting of hundreds of spatulae on each seta. It is the van der Waals forces between these fine structures and the surface which produce the high adhesion and friction forces between gecko and the surface [20]. High image resolution techniques have shown the hierarchical morphology that covers the skin on the gecko's foot (Fig. 2). The hierarchical structure of gecko skin which comprises lamella, setae, and spatulae allows gecko to attach to and detach from any kind of surface.

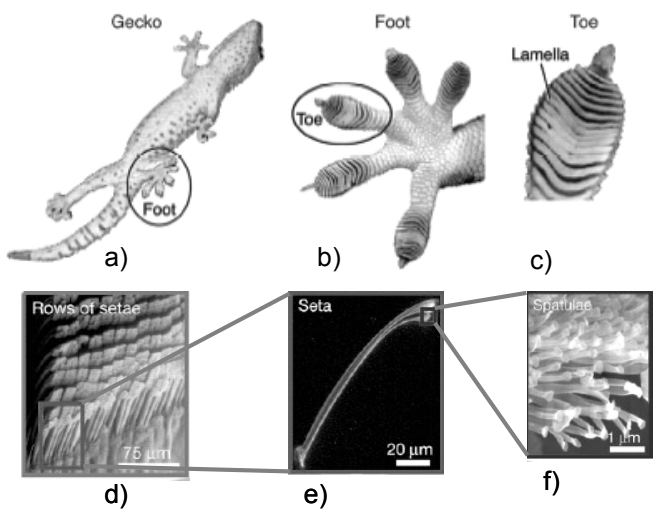

Figure 2: The hierarchical structure of geckos: a) gecko, b) foot, c) toe, d) rows of setae, e) seta and f) spatula pads [21,22].

\section{Non animal world}

Natural world has been constantly improved through evolution for billions of years. The living organisms have been undergoing a process of adaptation to enable them to explore new territories and cope with new environmental conditions. Through this process some interesting frictional characteristics have been formed and some of them have been mentioned above. There are however some other natural systems where control of friction is not being part of their evolution but they still have potentially interesting properties from a technical point of view. These are mainly non living systems and two of them are discussed in more details in this section.

\subsection{Ice friction}

Ice is well known for its low friction properties and in fact it is characterised by the lowest coefficient of friction from all crystalline solids. This phenomena has 
been studied for more than 100 years [23] and it is widely accepted that the frictional behaviour of ice is a function of two physical mechanisms: water lubrication and adhesion, which are strongly dependent on the environment temperature and sliding velocity. The water lubrication mechanism was proposed by Bowden and Hughes in late 30's [24], to explain dependence of friction on the sliding velocity. It has been found that for a given load and temperature around $263 \mathrm{~K}$, water lubrication mechanism takes place at velocity above $0.01 \mathrm{~ms}^{-1}$ and adhesion mechanism below that velocity. It was related to the amount of energy generated at the interface from frictional heating viscous behaviour of the water film. More recently the ice/slider interface has been studied by means of low-temperature scanning electron microscopy to reveal the morphology of wear tracks and detailed friction map has been plotted as a function of sliding velocity and the environment temperature [25]. An important characteristic of the ice surface has been established when a thin liquid-like layer was discovered below the melting point of ice [26]. To study that phenomena in more detail a research group from France used a sophisticated tribometer to measure friction at temperatures as low as $123 \mathrm{~K}$ [27]. From that work equilibrium and non- equilibrium phases have been identified as a function of the amount of frictional energy provided.

Fundamental research on ice friction has practical applications in the field of various structural materials being in contact with ice, like skies, skates or tyres but helps also to understand a water lubrication mechanism, which has a potential to become a novel, environmentally-friendly technology.

\subsection{Mucilage for lubrication purposes}

Mucilage is a gelatinous substance secreted by nearly all classes of plants and some microorganisms, comprised usually of protein, polysaccharides and uranides. Its main functions are to act as a membrane thickener, help with seed germination, aid in water storage and provide food reserve. However it is not primarily designed by nature to enhance frictional behaviour of plants, it is a very interesting example of using an organic material for lubrication purposes. This field has not been studied intensively, nevertheless it has been found that mucilage and mucilage extracts can act as an effective waterbased, environmentally friendly, non-toxic lubricants or coolants [28]. It has been postulated also that plant mucilage could be used for hydrodynamic drag reduction on the outer hull of watercraft [28]. The tribological characteristics of aloe mucilage has been recently verified on some metallic components [29], suggesting beneficial impact and potential future use of this bio-lubricant.

One of the medium-sized trees native to North America is called slippery elm (Ulmus Fulva), which suggest some superior frictional properties related to that plant. Indeed, a mucilage extracted from a bark of slippery elm has remarkable lubricating properties and is commercially available as a remedy, which has a very strong soothing effect on the digestive tract by building a coating on he surface of the gastrointestinal lining allowing matter to pass easily [30]. 


\section{Biomimetics and bioinspiration in tribology}

We have shown some exciting examples of how nature optimises frictional behaviour in the animal world and how nature deals with friction in case of non animals. The role of the engineer is firstly to recognise potential in a natural system, and secondly to apply derived principles in practice to enhance mechanical performance of a technical system. In that respect surface engineering is a tool, which can enable the transition from a natural system to the engineered one to be made by mimicking natural system through manipulation of surface properties of the component.

\subsection{Shark skin}

It is currently agreed that low hydrodynamic drag of shark skin is achieved by a number of factors but it has been also recognised that the most important one is a specific morphology of the skin. Hence, an effort has been undertaken to mimic the skin morphology by using an appropriate surface engineering techniques.

The most spectacular application of shark skin principle is probably related to swimsuit and wetsuit surfaces. It was widely discussed during the 2000 Olympics where most of the swimmers wore the Speedo Fastskin FSII suits to reduce drag by $4 \%$ [31]. The greater efficiency of the suit was achieved by application of computational fluid dynamics to model water flow along groves so that microscopic vortices were produced on the fabric surface reducing the hydrodynamic drag.

Other research revealed that micro-riblets can be applied on the inner surfaces of pipes to speed flow of water by up to $10 \%$ [32]. It has been also found that structures similar to the shark skin morphology created on the plastic film attached to the aircraft surface reduces aerodynamic drag and can cut fuel consumption by $1 \%$ [33]. Finally shark-like riblets were used successfully on the winning yacht in the 1987 America's Cup races and were believed to offer such an advantage that are now prohibited from competition [34].

\subsection{Gecko-inspired adhesives}

The physical aspect of the adhesion mechanism used by gecko makes this mechanism very desirable for technology applications. As shown in section 2.3 the key component of gecko's adhesion are the intermolecular van der Waals forces. In order for these forces to ensure good adhesion, a large amount of intimate surface contact to the binding surface is needed. In gecko, this is achieved by a multilevel conformal system consisting lamella, setae and spatulae which provide surface conformation in a broad range of scale. The first step towards developing gecko-inspired adhesion technologies will be to develop surfaces with micro and nano fibers able to provide high surface conformity. As potential materials to form these fibers are considered to be polymers [35] and development of gecko-inspired surface climbing robots with these materials has been tested [36]. Formation of gecko-inspired adhesive tapes has also been shown with the carbon nanotubes [37] attached on a flexible polymer tape. This 
tape is shown to be more effective than the tape formed with polymers pillars, withstanding up to ten times higher shear forces than polymer pillars.

In general, the successful transfer of gecko adhesion mechanism into developing synthetic adhesive tapes in a large extend is depended on the fabrication processes to develop underlying gecko-inspired nanostructures. Further research should be aimed in developing fast and inexpensive fabrication processes, able to fabricate surfaces that show functionalities as the natural surfaces.

\section{Future challenges and conclusions}

Biomimetics is an exciting field for tribologists with many natural systems characterised by remarkable frictional properties. Potential of some of those systems has been appreciated decades ago, e.g. synovial joints, but new discoveries are emerging, e.g. mucilage applied for lubrication purposes, and many others remain still undiscovered. In order to be applied in the engineering environment, fundamental behavior of a natural system needs to be fully understood in the first instance and then mimicked by using an appropriate surface engineering technique. It is nowadays possible to apply novel emerging technologies to translate natural principles into engineering practice. The main challenge remains in imitating natural systems, which operates using soft materials and water based lubricants. We have to also remember that nature does not use simplistic solutions and each functional behavior is supported by a system approach, which is a result of interaction of several factors as unique material properties, hierarchical structure, specialized additives or sophisticated surface morphology.

\section{References}

[1] Bar-Cohen, Y., (ed). Biomimetics: biologically inspired technologies, Boca Raton, FL; London: Taylor \& Francis, 2006.

[2] Dowson, D., History of Tribology, London: Professional Engineering Pubs., 1998.

[3] Fisher J., Biomedical applications. Modern Tribology Handbook, ed. B. Bhushan, Vol. II, CRC Press LLC, 2001.

[4] Dumbleton J.H., Tribology of Natural and Artificial Joints, Elsevier: Amsterdam, 1981.

[5] Mow V.C., Holmes M.H. \& Lai W.M., Fluid transport and mechanical properties of articular cartilage: A review. J. Biomechanics, 17, pp. 377394, 1984.

[6] Mansour J.M., Biomechanics of cartilage (Chapter 5). Kinesiology: The Mechanics and Pathomechanics of Human Movement, ed. C.A. Oatis, Lippincott Williams and Wilkins: Philadelphia, 2003.

[7] Loret B. \& Simoes F.M.F., Articular cartilage with intra- and extrafibrillar waters: a chemo-mechanical. Mechanics of Materials, 36, pp. 515-541, 2004. 
[8] Krishnan, R., Kopacz, M. \& Ateshian, G.A., Experimental verification of the role of interstitial fluid pressurization in cartilage lubrication. Journal of Orthopaedic Research, 22, pp. 565-570, 2004.

[9] Schwarz I.M. \& Hills B.A., Surface-Active Phospholipid as the Lubricating Component of Lubricin. British Journal of Rheumatology, 37, pp. 21-26, 1998.

[10] Hills B.A., Surface-active phospholipid: a Pandora's box of clinical applications. Part II. Barrier and lubricating properties. Internal Medicine Journal, 32, pp. 242-251, 2002.

[11] Kobayashi, A., Yamamoto, I. \& Aoyama, T., Tribology of a snail (terrestrial gastropod). Proc. of the $29^{\text {th }}$ Leeds-Lyon Symposium on Tribology, Elsevier B.V., Tribology Series, 41, pp. 429-436, 2004.

[12] Lauga E. \& Hosoi A.E., Tuning gastropod locomotion: Modeling the influence of mucus rheology on the cost of crawling. arXiv:condmat/0608363v2 [cond-mat.soft]

[13] Federle, W., Barnes, W.J.P., Baumgartner, W., Drechsler, P. \& Smith, J.M., Wet but not slippery: boundary friction in tree frog adhesive toe pads. Journal of the Royal Society Interface, 3(10), pp. 689-97, 2006.

[14] Ling, S.C. \& Ling T.Y.J., Anomalous drag-reducing phenomenon at a water/fish-mucus or polymer interface. J. Fluid Mech., 65(3), pp. 499-512, 1974.

[15] Jakowska, S., Mucus secretion in fish - a note. Ann. N. Y. Acad. Sci. 160, 458-462, 1963.

[16] Daniel, T.L., Fish mucus: In situ measurements of polymer drag reduction. Biol. Bull., 160, pp. 376-382, 1981.

[17] Meyers, M.A., Lin, A.Y.M., Seki, Y., Chen, P.-Y., Kad, B.K. \& Bodde, S., Structural biological composites: An overview. JOM, pp. 36-43, 2006.

[18] Barnes, W.J.P., Biomimetic solutions to sticky problems. Science, 318, pp. 203-204, 2007.

[19] Gorb, S.N., Functional surfaces in biology: Mechanisms and Applications. Biomimetics - Biologically inspired technologies, ed. Y. Bar-Cohen, Taylor \& Francis: London, pp. 381-398, 2006.

[20] Autumn, K., Sitti, M., Liang, Y.A., Peattie, A.M., Hansen, W.R., Sponberg, S., Kenny, T.W., Fearing, R., Israelachvili, J.N. \& Full, R.J., Evidence for van der Waals adhesion in gecko setae. Proc Natl Acad Sci, 99(19), pp. 12252-12256, 2002.

[21] Autumn, K., Liang, Y.A., Hsieh, S.T., Zesch, W., Chan, W.P., Kenny, T.W., Fearing, R. \& Full, R.J., Adhesive force of a single gecko foot-hair. Nature, 406, pp. 681-685, 2000.

[22] Tian, Y., Pesika, N., Zeng, H., Rosenberg, K., Zhao, B., McGuiggan, P., Autumn, K. \& Israelachvili, J., Adhesion and friction in gecko toe attachment and detachment. Proc Natl Acad Sci, 103(51), pp. 1932019325, 2006.

[23] Reynolds, O., On the slipperiness of ice. Mem. And Proc. Manch. Lit. Phil. Soc., 43, pp. 1-7, 1900. 
[24] Bowden, F.P. \& Hughes, T.P., The mechanisms of sliding on ice and snow. Proc. R. Soc. London, Ser. A, 172(949), pp. 280-298, 1939.

[25] Marno, B.A., Blackford, J.R. \& Jeffree, C.E., Ice friction, wear features and their dependence on sliding velocity and temperature. Journal of Glaciology, 51(174), pp. 391-398, 2005.

[26] Petrenko, V.F., Physics of ice, Oxford University Press: New York, 1999.

[27] Liang, H., Martin, J.M. \& Le Mogne, T., Friction-induced nonequilibrium phase transformation of low-temperature ice. Journal of Applied Physics, 97, 043525, pp. 1-5, 2005.

[28] Organic lubricants and coolants, United States Patent 6124248.

[29] Xu, J., Luo, J., Liu, S., Xie, G. \& Ma, L., Tribological characteristics of aloe mucilage. Tribology - Materials, Surfaces \& Interfaces, article in press.

[30] University of Maryland Medical Center, http://www.umm.edu/altmed/ articles/slippery-elm-000274.htm\#Plant\%20Description

[31] Curtis, D. \& Carre, M., Olympians look to physics. Physics World, 17(8), pp. 19-20, 2004.

[32] Koeltzsch, K., Dinkelacker, A. \& Grundmann R., Flow over convergent and divergent wall riblets. Experiments in Fluids, 33(2), pp. 346-350, 2002.

[33] Viswanath, P.R., Aircraft viscous drag reduction using riblets. Progress in Aerospace Sciences, 38(6-7), pp. 571-600, 2002.

[34] Krieger, K., Do Pool Sharks Swim Faster? Science, 305(5684), pp. 636637, 2004.

[35] Northern, M.T. \& Turner, K.L., A batch fabricated biomimetic dry adhesive. Nanotechnology, 16, pp. 1159-1166, 2005.

[36] Murphy, M.P. \& Sitti, M., Waalbot: An agile small-scale wall-climbing robot utilizing dry elastomer adhesives. IEEE/ASME Transactions on mechatronics, 12(3), pp. 330-338, 2007.

[37] Ge, L., Sethi, S., Ci, L., Ajayan, P.M. \& Dhinojwala, A., Carbon nanotubebased synthetic gecko tapes. Proc Natl Acad Sci, 104(26), pp. 1079210795, 2007. 